\title{
Optimization of extraction parameters for the chemical profiling of 3,4-methylenedioxymethamphetamine (MDMA) tablets
}

\author{
Pascal Gimeno, Fabrice Besacier*, \\ Huguette Chaudron-Thozet \\ Laboratoire de Police Scientifique de Lyon, 31 Avenue Franklin Roosevelt, 69134 Ecully, France
}

Received 9 October 2002; received in revised form 6 January 2003; accepted 10 January 2003

\begin{abstract}
The extraction of impurities from illegally produced 3,4-methylenedioxymethamphetamine (MDMA) has been studied in order to optimize the parameters. Two different MDMA samples were used. Particular attention was paid to the influence of the $\mathrm{pH}$, the evaporation step, and the sample storage. The method used was an extraction of impurities by diethyl ether from a buffer solution at $\mathrm{pH} 11.5$, followed by gas chromatography (GC) mass spectrometric (MS) analyses after a dryness concentration under monitored conditions of the ethereal extract. Repeat extractions of the same sample gave an average relative standard deviation (RSD) of less than $8.5 \%$ within day and less than $10.5 \%$ between days.
\end{abstract}

(C) 2003 Elsevier Science Ireland Ltd. All rights reserved.

Keywords: 3,4-Methylenedioxymethamphetamine (MDMA); Impurities; Gas chromatography; Mass spectrometry; Profiling

\section{Introduction}

3,4-Methylenedioxymethamphetamine (MDMA) is an illicit synthetic, psychoactive substance possessing stimulant and mild hallucinogenic properties. According to Europol, in 2000, 17.4 millions of ecstasy tablets were seized in the member states of the European Union, corresponding to an increase of almost 50\% compared with 1999. Significant increases were observed in Austria (420\%), Finland (394\%), Greece (1803\%), Ireland (163\%), Italy (86\%), The Netherlands (50\%), Spain (64\%) and Sweden (152\%) [1].

In order to know synthesis schemes used by clandestine laboratories, an analytical method has been developed in order to identify by gas chromatography-mass spectrometry (GC-MS) the various impurities present in ecstasy samples [2]. Nevertheless, several extraction parameters needed to be optimized in order to improve the reproducibility of the method suggested.

As a matter of fact, if many publications deal with a detailed impurity extraction process for the profiling of

\footnotetext{
*Corresponding author. Tel.: +33-47-286-8982.

E-mail address: fabrice.besacier@interieur.gouv.fr (F. Besacier).
}

amphetamine or methamphetamine samples like the paper of Sten et al. [3], only few articles are concerned with MDMA [4-7]. More authors prefer to focus on the identification of impurities in freshly prepared MDMA samples via different synthesis routes and give us analytical data of precursors, intermediates and reaction by products [8-18]. Among published extraction processes, one consists in dissolving $5 \mathrm{mg}$ of crushed MDMA tablets into $1 \mathrm{ml}$ of redistilled diethyl ether [4]. The supernatant is then taken off and evaporated to dryness before adding $0.1 \mathrm{ml}$ of methyl alcohol for GC-MS analyses. Another paper presents the impurities found in MDMA and MDEA street samples [5]. The extraction method used consists in dissolving 150-300 $\mathrm{mg}$ of each sample into $5 \mathrm{ml}$ of phosphate buffer $(\mathrm{pH}=7)$, in order to have about $80 \mathrm{mg}$ of active substance, the extraction being carried out with $1 \mathrm{ml}$ of diethyl ether containing heneicosane (C21) as internal standard. Other authors also use a phosphate buffer $(\mathrm{pH}=6[6]$ or $\mathrm{pH}=9$ [7]) to dissolve MDMA powders whereas organic impurities are extracted, respectively by dichloromethane [6] and ethyl acetate [7]. In that last study, comparison between liquid-liquid extraction (LLE) and solid phase extraction (SPE) for the profiling of ecstasy tablets is also discussed. 


\section{Materials and methods}

\subsection{Gas chromatography and mass spectrometry}

All analyses were carried out on a Thermofinnigan GC trace 2000 gas chromatograph interfaced with an ion trap Polaris mass spectrometer. Two microliters of each extract were injected according to the splitless mode using a Thermofinnigan AS 2000 autosampler. The column was a Supelco PTA5 capillary column (cross-linked poly 5\% diphenyl/95\% dimethylsiloxane); $\quad 30 \mathrm{~m} \times 0.32 \mathrm{~mm}($ i.d. $) \times 0.5 \mu \mathrm{m} \quad$ film thickness. The oven temperature was programmed as follows: $50{ }^{\circ} \mathrm{C}$ for $1 \mathrm{~min}, 5{ }^{\circ} \mathrm{C} \mathrm{min}{ }^{-1}$ to $150{ }^{\circ} \mathrm{C}$ for $12 \mathrm{~min}$, and $15^{\circ} \mathrm{C} \mathrm{min}{ }^{-1}$ to $300{ }^{\circ} \mathrm{C}$ for $10 \mathrm{~min}$. The injection port and transfer line temperatures were, respectively 280 and $275^{\circ} \mathrm{C}$. The ion source temperature was set at $200{ }^{\circ} \mathrm{C}$, and the helium carrier gas flow rate was fixed at $1 \mathrm{ml} \mathrm{min}^{-1}$. The mass spectrometer was tuned on electron impact ionization (Ei) for low-mass analysis for detection of each impurity. For the reproducibility and the optimization studies, selected ion monitoring (SIM) was used on the most intense impurity mass fragments. In order to preserve the MS filament life, the mass spectrometer was switched-off during elution of the major compounds.

\subsection{MDMA materials}

Two different MDMA samples (RefA and RefB) have been used for the optimization of extraction parameters. These samples consisted of 35\% MDMA Phosphate diluted with lactose (RefA), and of 99\% MDMA hydrochloride (RefB).

\subsection{Standard extraction method}

An amount of sample equivalent to $10 \mathrm{mg}$ of pure MDMA hydrochloride was weighed and dissolved into $2 \mathrm{ml}$ of a buffer solution at $\mathrm{pH} 11.5$ and shaken for $10 \mathrm{~min}$ at $1800 \mathrm{rpm}$. The extraction was performed adding $3 \mathrm{ml}$ of diethylether and shaking for another $10 \mathrm{~min}$. After centrifugation, the organic layer was transferred to a conic tube and evaporated to dryness under monitored conditions at room temperature (extracts were evaporated to dryness under a low nitrogen flow rate). Five hundred microliter of diethylether containing $n$-dodecane as ISTD at $0.113 \mathrm{ppm}$ were added to the tube, shaken for a few seconds, and transferred to a micro-vial for profile analysis. In order to avoid impurity degradation, the extracts were injected the same day they were prepared.

\section{Results and discussion}

\subsection{Identification of impurities}

The chromatographic profiles of samples RefA and RefB are shown in Figs. 1 and 2, respectively. Table 1 gives peak identity and mass spectral data for impurities used in this study. Target ions used in the SIM mode are bold typed in the table.

\subsection{Overall reproducibility of the method}

Results were expressed giving relative standard deviation (RSD) of each peak area, acquired according to SIM mode and after normalization, i.e. dividing all areas in a run by the peaks sum. Peaks used for this study were peaks $1-10$, for both samples RefA and RefB (Figs. 1 and 2). However, impurity 6 is not present in sample RefB.

\subsubsection{Gas chromatography repeatability}

Five injections of the same extract from sample RefA gave a minimum relative standard deviation of $1.8 \%$ to a maximum of $7.0 \%$, the average value being $4.9 \%$. The same study on sample RefB gave a minimum relative standard deviation of $0.9 \%$ to a maximum of $9.3 \%$, the average value being $6.1 \%$.

\subsubsection{Overall reproducibility (extraction and gas chromatography)}

3.2.2.1. Within day. Four extractions by day during 4 days were made from samples RefA and RefB and analyzed. The relative standard deviations for RefA sample varied from 3.5

Table 1

Target impurities in MDMA samples

\begin{tabular}{|c|c|c|}
\hline Impurity name & Ei mass spectral data & Peak no. \\
\hline 1,3-Benzodioxole $\mathrm{C}_{7} \mathrm{H}_{6} \mathrm{O}_{2} ;$ MW 122 & 121/122, 63/64 & 1 \\
\hline 3,4-Methylenedioxytoluene $\mathrm{C}_{8} \mathrm{H}_{8} \mathrm{O}_{2} ; \mathrm{MW} 136$ & 135/136, 78/77, 51 & 2 \\
\hline Safrole $\mathrm{C}_{10} \mathrm{H}_{10} \mathrm{O}_{2} ; \mathrm{MW} 162$ & $\mathbf{1 6 2}, 104,131,77,51$ & 3 \\
\hline Piperonal $\mathrm{C}_{8} \mathrm{H}_{6} \mathrm{O}_{3}$; MW 150 & $\mathbf{1 4 9} / 150,121,63,91$ & 4 \\
\hline Isosafrole $\mathrm{C}_{10} \mathrm{H}_{10} \mathrm{O}_{2} ; \mathrm{MW} 162$ & $\mathbf{1 6 2}, 104,131,77,51$ & 5 \\
\hline 3,4-Methylenedioxy- $N$-methylbenzylamine $\mathrm{C}_{9} \mathrm{H}_{11} \mathrm{NO}_{2}$; MW 165 & $\mathbf{1 3 5} / 136,164 / 165,44,77$ & 6 \\
\hline p-Methoxymethamphetamine ( $p$ MMA) $\mathrm{C}_{11} \mathrm{H}_{17} \mathrm{NO}$; MW 179 & $\mathbf{5 8}, 121,78,91$ & 7 \\
\hline 1,2-Methylenedioxy-4-(2- $N$-methyliminopropyl)benzene $\mathrm{C}_{11} \mathrm{H}_{13} \mathrm{NO}_{2} ;$ MW 191 & 56, $191,135,77$ & 8 \\
\hline$N, N$-Dimethyl-(1,2-methylenedioxy)-4-(2-aminopropyl)benzene $\mathrm{C}_{12} \mathrm{H}_{17} \mathrm{NO}_{2} ; \mathrm{MW} 207$ & $\mathbf{7 2}, 56,44,73,58,70$ & 9 \\
\hline$N$-Methyl-(1,2-methylenedioxy)-4-(1-ethyl-2-aminopropyl)benzene $\mathrm{C}_{13} \mathrm{H}_{19} \mathrm{NO}_{2} ; \mathrm{MW} 221$ & $58, \mathbf{1 6 2}, 77,135,194$ & 10 \\
\hline
\end{tabular}



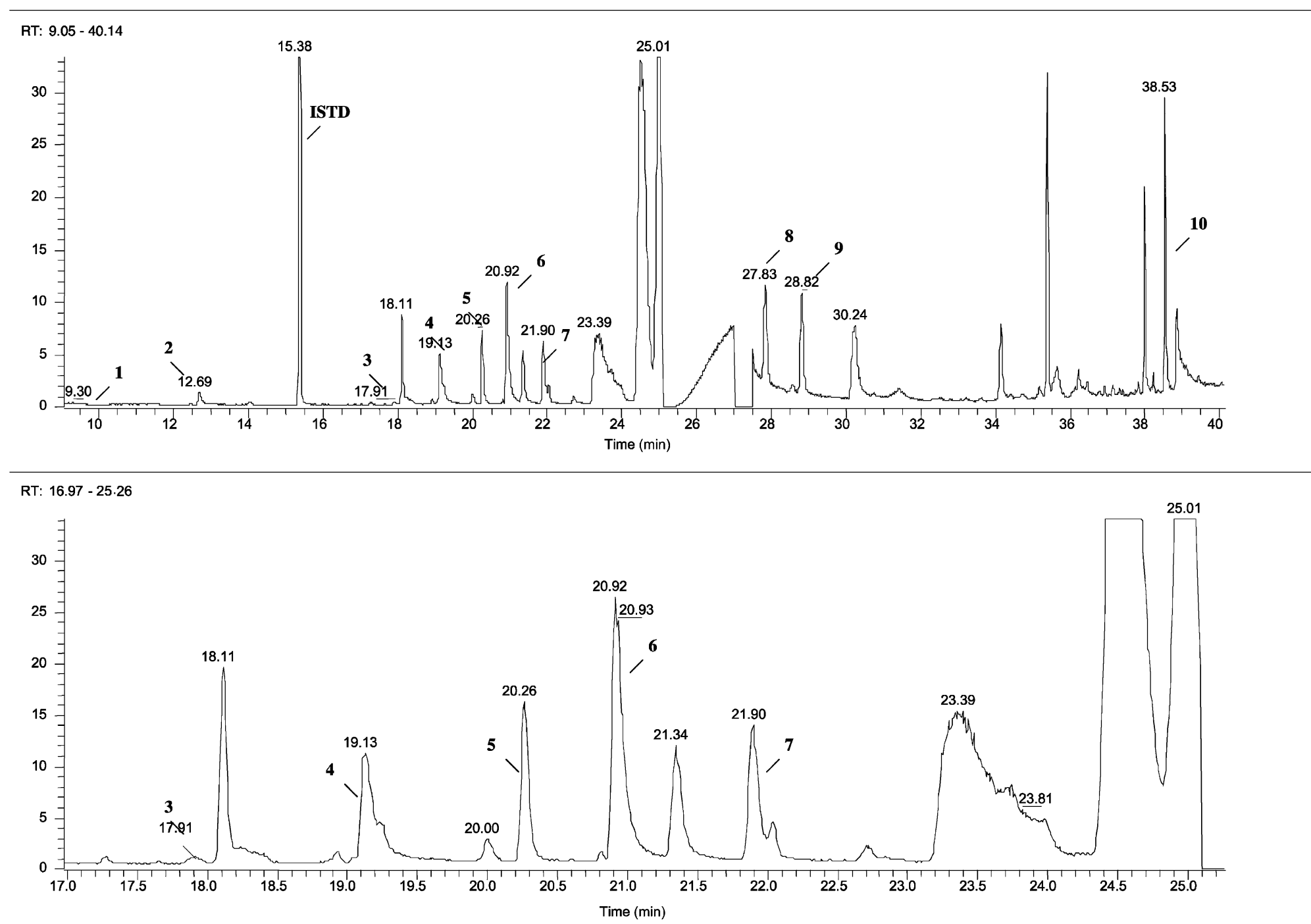

Fig. 1. Ei/full scan impurity profile of sample RefA. 

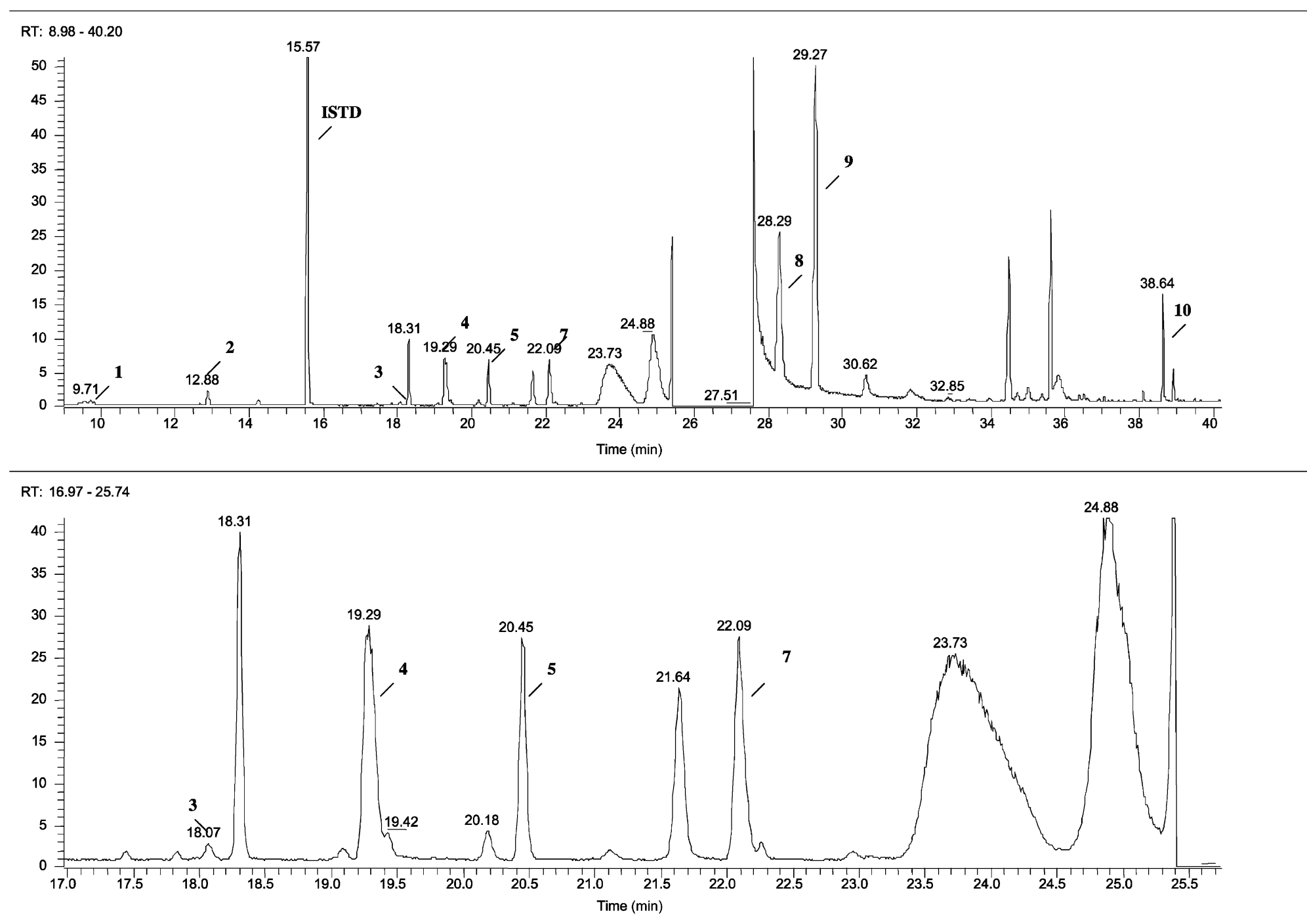

Fig. 2. Ei/full scan impurity profile of sample RefB. 
Table 2

Within day repeatability (RSD\%)

\begin{tabular}{llllllllllll}
\hline Sample/peak & 1 & 2 & 3 & 4 & 5 & 6 & 7 & 8 & 9 & 10 & Average \\
\hline RefA & 9.2 & 6.4 & 7.1 & 13.3 & 8.7 & 3.5 & 5.4 & 9.4 & 7.8 & 6.3 & 7.7 \\
RefB & 10.3 & 8.1 & 11.0 & 10.3 & 7.5 & & 6.9 & 4.6 & 8.7 & 5.7 & 8.1 \\
\hline
\end{tabular}

Table 3

Between days reproducibility (RSD\%)

\begin{tabular}{llllllllllll}
\hline Sample/peak & 1 & 2 & 3 & 4 & 5 & 6 & 7 & 8 & 9 & 10 & Average \\
\hline RefA & 12.3 & 8.4 & 6.6 & 16.3 & 10.9 & 6.9 & 7.2 & 12.4 & 13.0 & 7.4 & 10.2 \\
RefB & 12.1 & 9.9 & 10.9 & 10.6 & 10.5 & & 8.9 & 7.2 & 11.1 & 8.2 & 9.9 \\
\hline
\end{tabular}

to $13.3 \%$ with an average of $7.7 \%$, and similar results were obtained for RefB sample with a minimum relative standard deviation of $4.6 \%$ to a maximum of $11.0 \%$ and an average of $8.1 \%$. Table 2 gives the results obtained for each target impurity.

3.2.2.2. Between days. Four extractions by day during four days were made from samples RefA and RefB and analyzed. If we consider sample RefA, the relative standard deviations varied from 6.6 to $16.3 \%$ depending on the impurity, with an average value of $10.2 \%$. For RefB sample, values varied from 7.2 to $12.1 \%$ with an average of $9.9 \%$. Table 3 gives the results obtained for each target impurity.

\subsection{Optimization of extraction parameters}

\subsubsection{Influence of the $\mathrm{pH}$}

A buffer solution of glycocoll- $\mathrm{NaCl} / \mathrm{NaOH}$ was used for the $\mathrm{pH}$ study. The $\mathrm{pH}$ was changed from 8.4 to 12.6 in increments of 0.2 . Results point out that almost all target impurities were strongly influenced by the buffer $\mathrm{pH}$. The extracted impurity amounts increased with the $\mathrm{pH}$ from 8.4

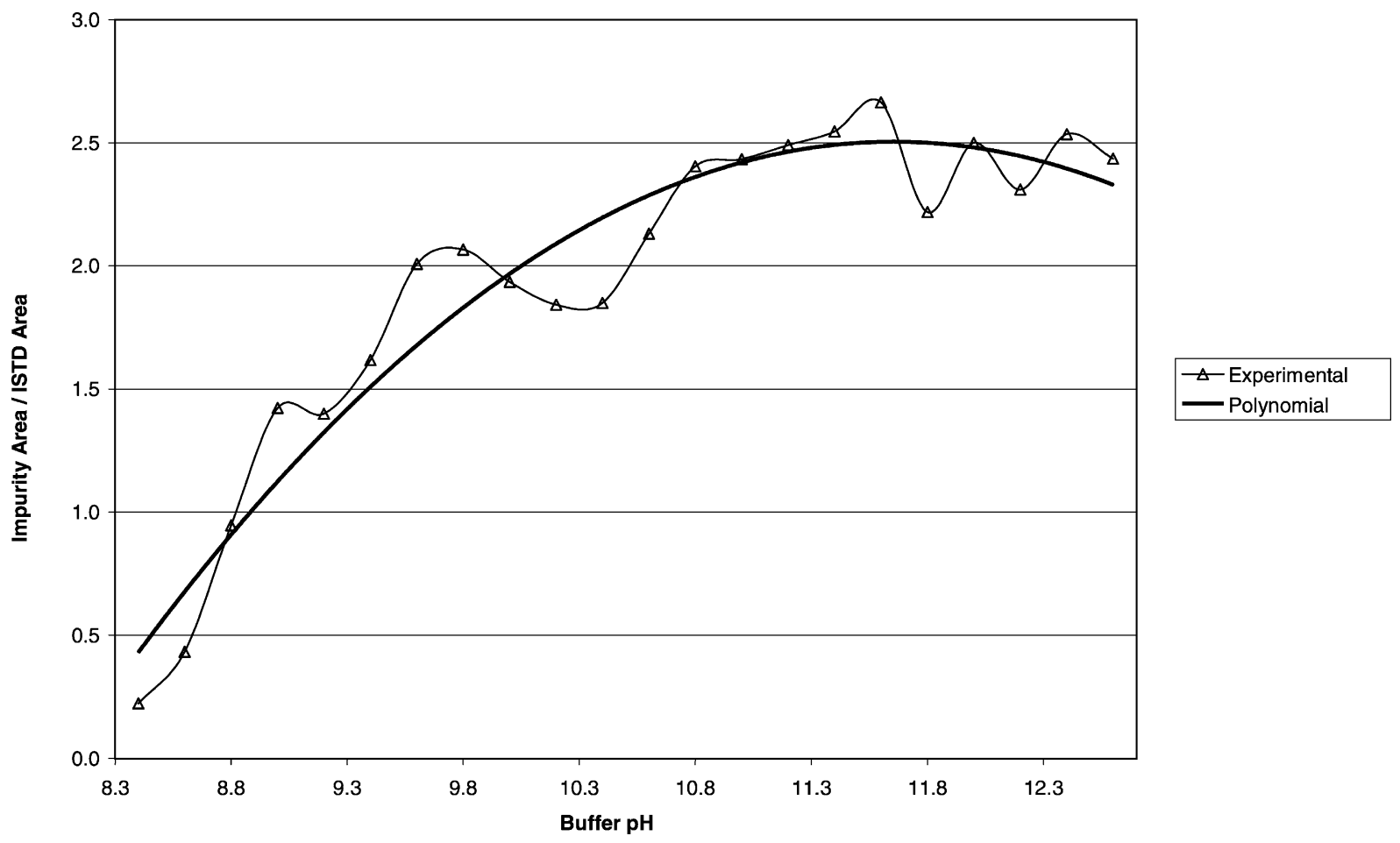

Fig. 3. Influence of $\mathrm{pH}$ on impurity 3. 


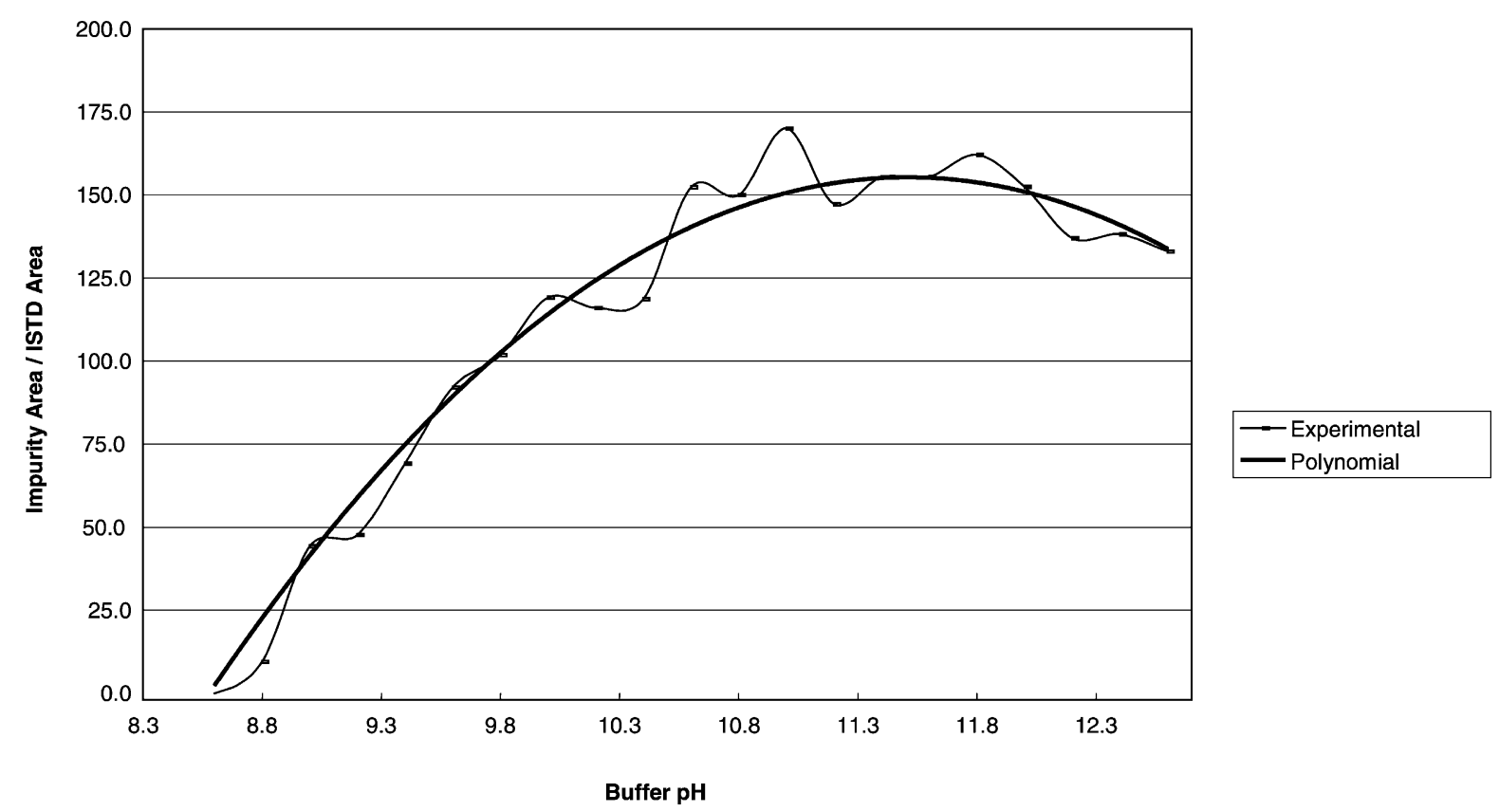

Fig. 4. Influence of pH on impurity 6.

Table 4

Relative standard deviations (calculated from the area ratio between each impurity and the ISTD) obtained for samples RefA and RefB extracted from buffer $\mathrm{pH} 10.8$ to 12.0

\begin{tabular}{lllllrlrrrrr}
\hline Sample/peak & 1 & 2 & 3 & \multicolumn{1}{l}{4} & \multicolumn{1}{c}{5} & 6 & 7 & 8 & 9 & 10 & Average \\
\hline RefA & 10.9 & 5.9 & 11.8 & 13.1 & 9.3 & 5.0 & 4.7 & 8.6 & 5.2 & 2.0 & 7.6 \\
RefB & 14.0 & 6.6 & 10.2 & 9.6 & 10.5 & & 4.9 & 11.2 & 5.3 & 5.9 & 9.1 \\
\hline
\end{tabular}

to $10.5-11.0$ where a maximum was reached. Further increase only slightly improved the extracted amounts. For instance, results obtained for peaks 3 and 6 of the sample RefA (corresponding respectively to the lower and the higher impurity peak area) for a $\mathrm{pH}$ range from 8.6 to 12.6 are plotted in Figs. 3 and 4.

Moreover, the relative standard deviations resulting from extractions between $\mathrm{pH} 10.8$ to 12.0 were not significantly higher than in the within day study (Table 4). Therefore, a buffer $\mathrm{pH}$ of 11.5 was chosen and small variations due to, for instance, buffer storage could be accepted.

\subsubsection{Influence of the extraction solvent}

Five different solvents were tested at the optimum buffer $\mathrm{pH}$ (11.5), for the extraction of impurities from sample RefA: diethyl ether, chloroform, cyclohexane, butyl alcohol and toluene. The extraction was processed as follows: an amount of sample equivalent to $10 \mathrm{mg}$ of pure MDMA hydrochloride was weighed and dissolved into $2 \mathrm{ml}$ of a buffer solution (pH 11.5) and shaken for $10 \mathrm{~min}$ at $1800 \mathrm{rpm}$.
Five hundred microliter of the extraction solvent were then added and shaken for $10 \mathrm{~min}$. After centrifugation, the organic layer was transferred to a micro-vial, and $2 \mu \mathrm{l}$ of the extracts were injected.

The normalized impurity areas obtained for each solvent are shown in Fig. 5. As we can see, diethyl ether seems to better extract low amount impurities (peaks 2, 4, 5) than other solvents such as butyl alcohol or toluene, even if impurity 10 does not have a good extraction yield. Impurity 8 was not considered because with some solvents (chloroform and toluene) the chromatographic peak was eluted with MDMA.

\subsubsection{Influence of the shaking times}

Three different shaking times $(5,10$, and $20 \mathrm{~min})$ were tested for the dissolution and the extraction steps for sample RefA. Results point out the necessity to shake the tube at least $10 \mathrm{~min}$ at each step in order to optimize both processes. Increasing the shaking times up to 20 min only slightly improved the results, therefore $10 \mathrm{~min}$ was chosen as the best compromise. 


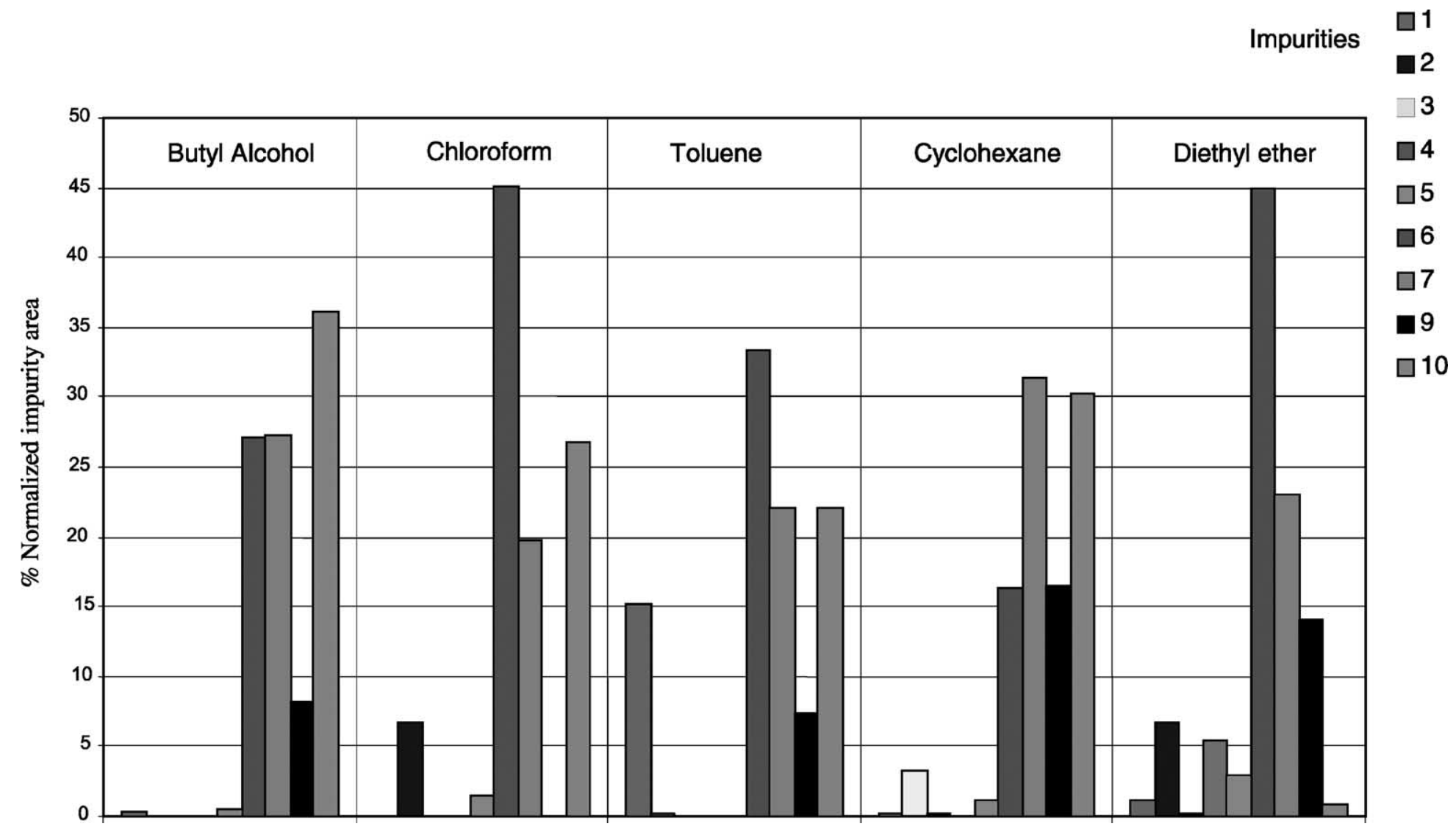


Table 5

Distribution of impurities between extraction phases

\begin{tabular}{lll}
\hline Diethyl ether $(\mathrm{ml})$ & Buffer $(\mathrm{ml})$ & Ratio \\
\hline 2 & 3 & $2 / 3$ \\
3 & 3 & 1 \\
3 & 2 & $3 / 2$ \\
\hline
\end{tabular}

\subsubsection{Distribution of impurities between extraction phases}

In order to evaluate the distribution of impurities between diethyl ether and the buffer solution at $\mathrm{pH} 11.5$, the amounts of diethyl ether and buffer were varied, while keeping the amount of samples (10 $\mathrm{mg}$ of pure MDMA) constant (Table 5). This evaluation was performed by direct comparison of the impurity area ratio (impurity area/ISTD area) depending on the volume ratios used.

As we can see in Fig. 6, some of the impurities $(2,8-10)$ have almost the same amounts extracted whatever is the ratio used, indicating that most of these impurities have been extracted. Others $(1,4-7)$ seem to depend more on the ratio between diethyl ether and the buffer, which indicates a lower extraction yield (Fig. 7). Therefore, the ratio $3 / 2$ was preferred.

\subsubsection{Influence of consecutive extractions}

Three consecutive extractions of samples RefA and RefB were made. Results point out that even after a third extrac- tion some MDMA impurities were still extracted. Fig. 8 shows the impurity amount extracted for the second and third extractions, compared to the first one for impurities 1 , 4,5 and 7. As expected, these impurities with low distribution ratios between diethyl ether and buffer are still present in the third extract. Nevertheless, other impurities $(2,3,6,8-$ 10) were no longer detected at the second extraction.

\subsubsection{Influence of solvent evaporation}

In order to investigate the influence of the evaporation step, four experiments involving three extractions each were performed on samples RefA and RefB. The first one consisted in evaporating extracts to dryness under a minimum nitrogen flow rate and stopping it right after complete evaporation (45 min approximatively). The second serie was performed using the same evaporation conditions but with a fixed time of $1 \mathrm{~h}$ and $45 \mathrm{~min}$ in order to study a possible impurity degradation. The third and fourth experiments had the same differences (changeable time VS fixed time) but with a high nitrogen flow (complete evaporation was performed in less than $10 \mathrm{~min}$ ).

First of all, no significant differences were observed between stopping or not the evaporation right after dryness. Therefore, it could be possible to use a longer evaporation time which allows not to look after the samples. Moreover, for all target impurities, the high evaporation speed increase the relative standard deviations obtained (Fig. 9 points out this influence for the sample RefA). Therefore, a slow rate has to be preferred.
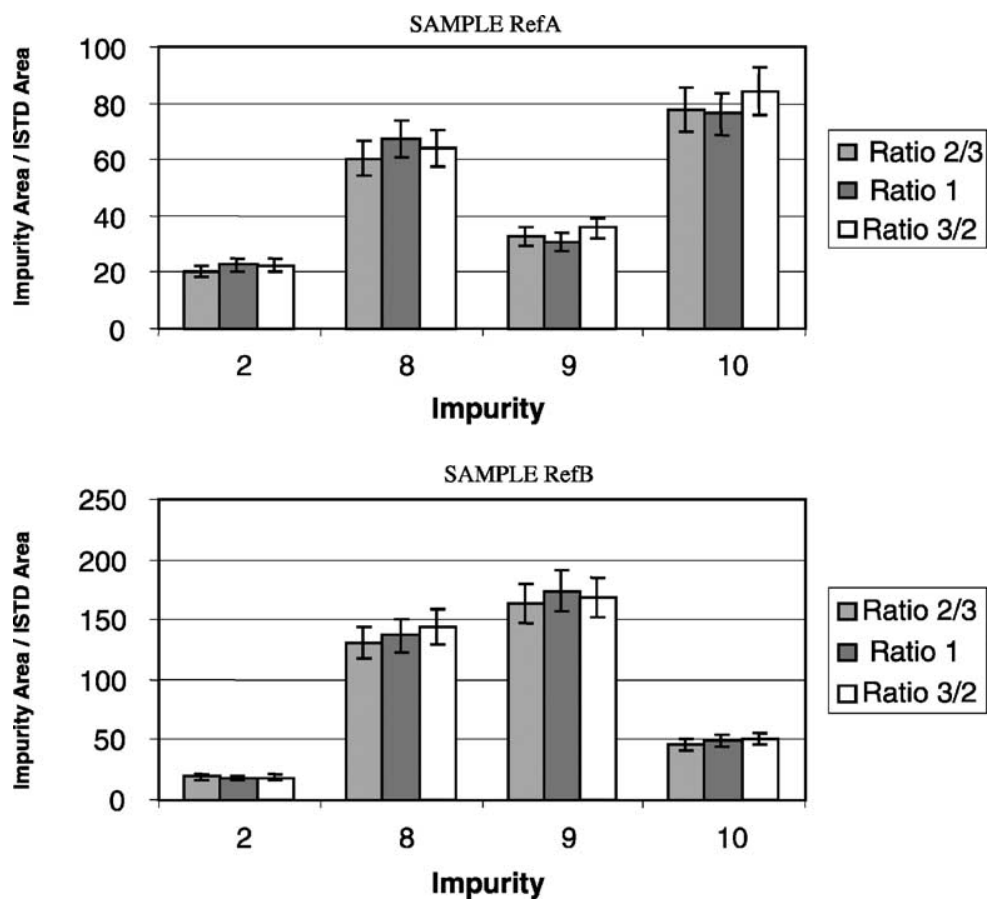

Fig. 6. Distribution of impurities 2, 8-10 between extraction phases (samples RefA and RefB). 

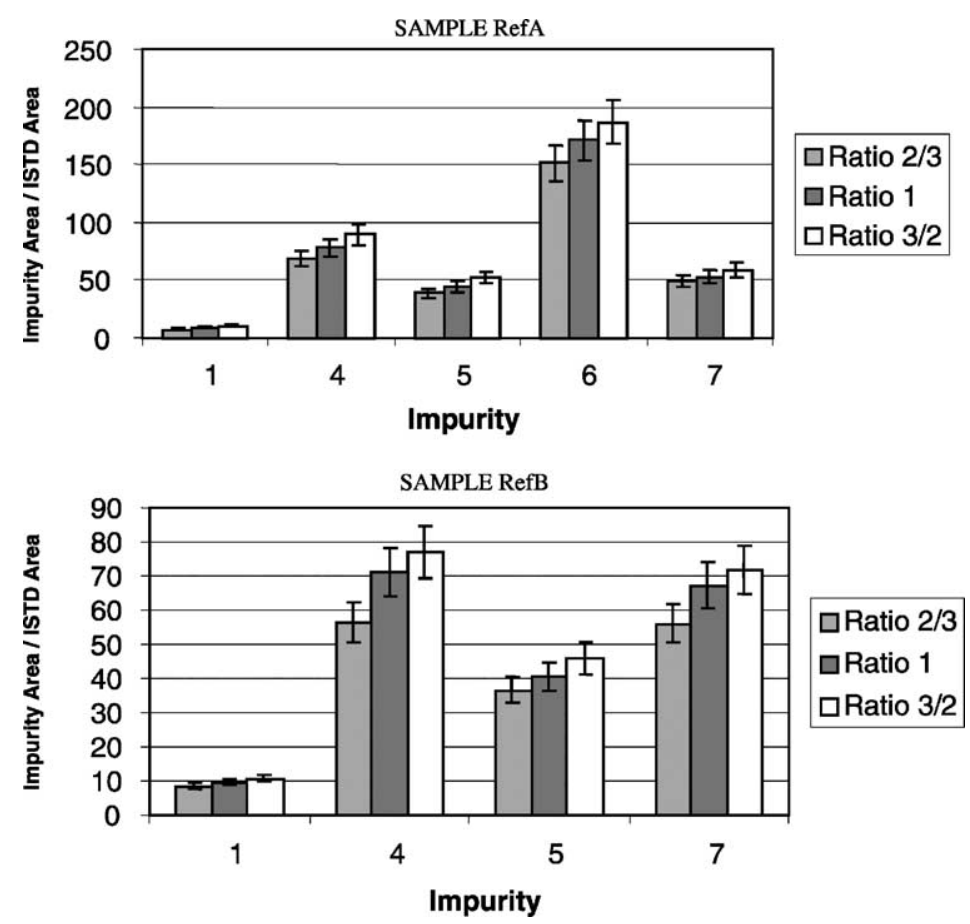

Fig. 7. Distribution of impurities 1, 4-7 between extraction phases (samples RefA and RefB).

\subsubsection{Storage of an extract}

As the extraction process and the run time analysis (50 $\mathrm{min}$ ) are quite long, they could lead to prepare numerous extracts and store them before analyses. In order to determine the best conditions to store the extracts, and to study the stability of impurities, three experiments were made on sample RefA. Two extracts were stored in the dark and, respectively at $6{ }^{\circ} \mathrm{C}$ and at ambient temperature. The third extract was exposed to day light at ambient temperature. All three extracts were analyzed the same day they were prepared and after 1-3 days. An internal standard ( $n$-dodecane) was added to determine the stability of all impurities. The peak area ratios between each target impurity and the internal standard were calculated for each storage time relative to the initial ratios. Impurity peak 3 could not be considered in this study due to its very low amount in the sample. Fig. 10 shows us the stability of impurities for extracts stored in a refrigerator (in the dark and, respectively at $\left.6{ }^{\circ} \mathrm{C}\right)$.

Regardless of impurity and even storage conditions, dramatic loss was noticed after 2 days of storage for most impurities, apart from impurities 8 and 10 which did not show changes larger than expected from analytical errors. Therefore, the extracts need to be analyzed the same day they are prepared.

We can also notice an increase of impurity 2 regardless of storage conditions. As a matter of fact, this impurity $(3,4-$ methylenedioxytoluene) is the common fragment of all impurities, therefore a total degradation of other compounds might lead to its formation.

\subsubsection{Influences of sample size and addition of lactose}

To investigate the influences of sample size and addition of lactose, two series of extractions were performed on sample RefB. The first serie consisted of two experiments using different amounts (9-11 mg) of pure MDMA $\mathrm{HCl}$. The relative standard deviation were then calculated between 9-10 $\mathrm{mg}$ on one hand and $10-11 \mathrm{mg}$ on the other hand and compared to results obtained with the within day repeatability $(8.1 \%)$. The relative standard deviations obtained varied from 2.6 to $15.4 \%$ with an average of $8.7 \%$, pointing out no significant influence of size variation on the results.

The second serie was performed to evaluate how lactose, which is the most common diluent, influences the extraction of impurities. For this experiment, different amounts of lactose $(0,50,60,70,80$ to $90 \%)$ were added to sample RefB. Fig. 11 show the influence of lactose on the normalized impurity areas. The relative standard deviations varied from 5.4 to $16.6 \%$ with an average of $10.9 \%$. If we consider samples diluted with an amount of lactose from 0 to $70 \%$, the deviations decreased from 3.5 to $14.9 \%$, with an average of $7.4 \%$. As a matter of fact, amounts of lactose higher than $60 \%$ seem to influence the extraction of impurities 8-10 (Fig. 11). It is then necessary to apply the method to samples diluted with less than $60 \%$ of lactose. 


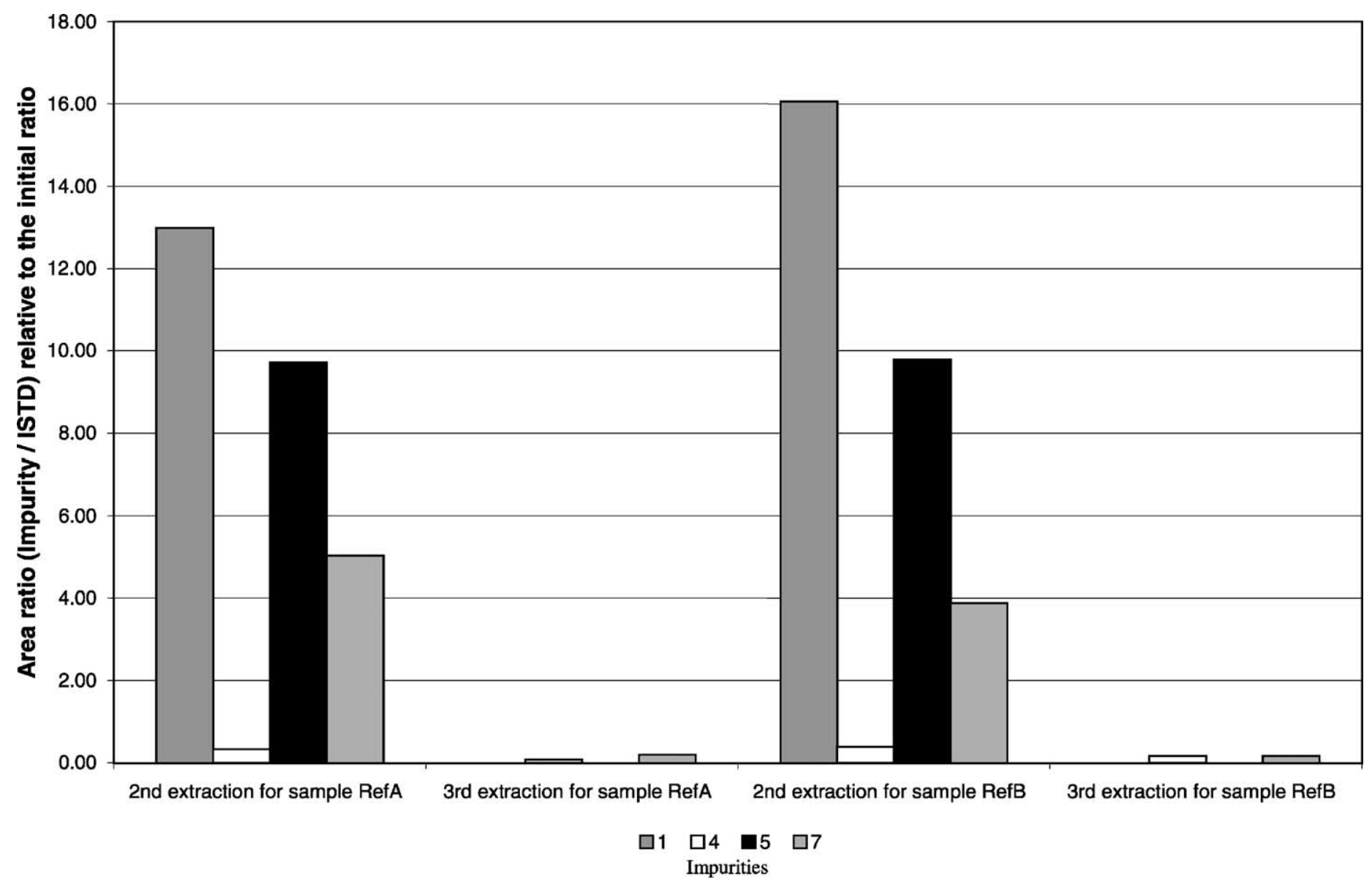

Fig. 8. Influence of consecutive extractions 


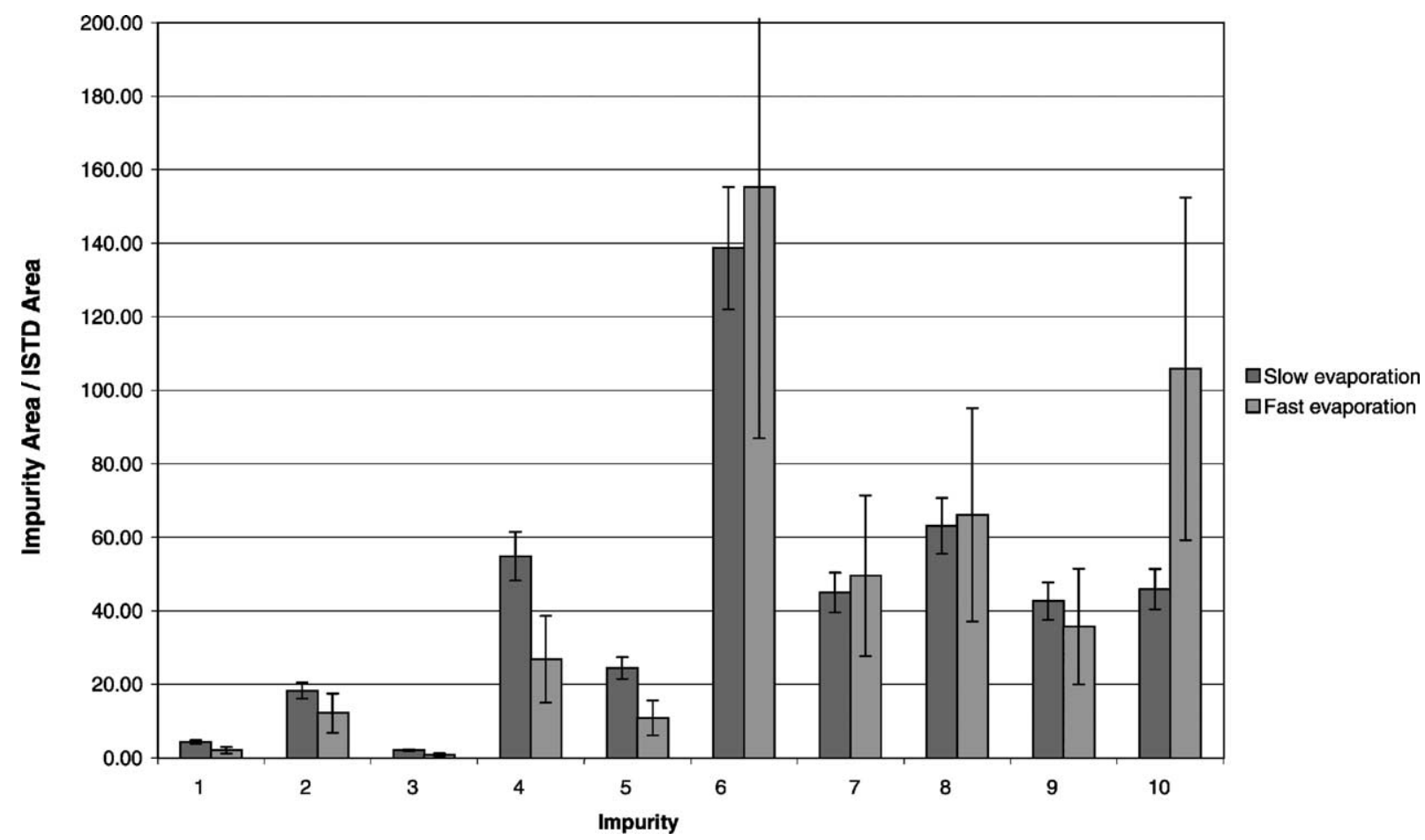

Fig. 9. Influence of solvent evaporation (sample RefA).

\subsubsection{Influence of volume variations}

Three solvent volumes were studied: buffer, extraction solvent (diethyl ether) and recovery solvent (diethyl ether). Table 6 shows the various volumes tested as well as the accuracy of the dispenser used. It can be noticed that the volume variation chosen were much larger than instrument accuracy. As a matter of fact, each variation corresponds to one graduation on the solvent dispenser that is for instance $0.1 \mathrm{ml}$ for the $5 \mathrm{ml}$ dispenser used in the buffer study.

Within each study, the normalized impurities areas and the relative standard deviations were calculated (Table 7). From a global point of view, only the recovery solvent volume has a significant influence on the results with an average RSD of $13.2 \%$ for sample RefA and $11.2 \%$ for sample RefB. However, volume variations of the other two

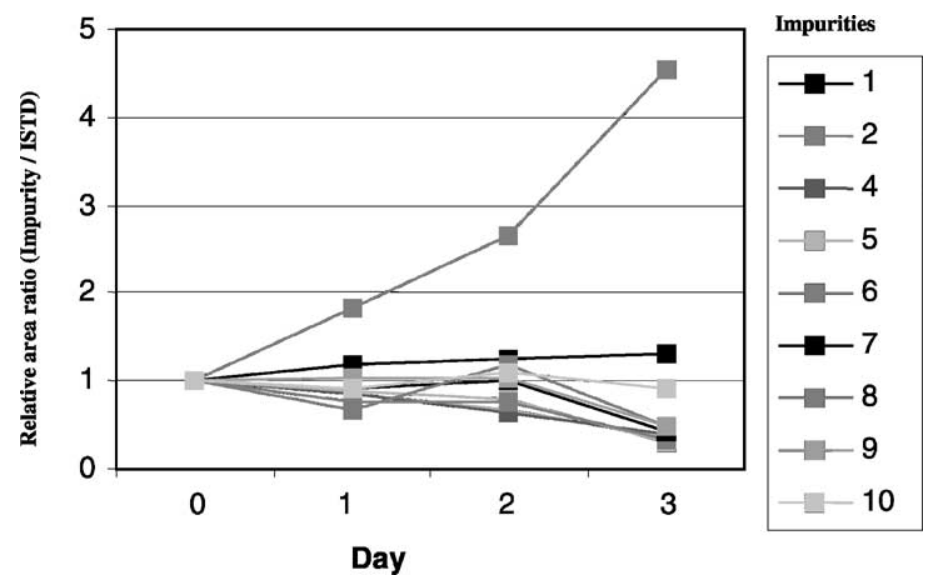

Fig. 10. Influence of storage conditions on the impurity stability (refrigerator). 

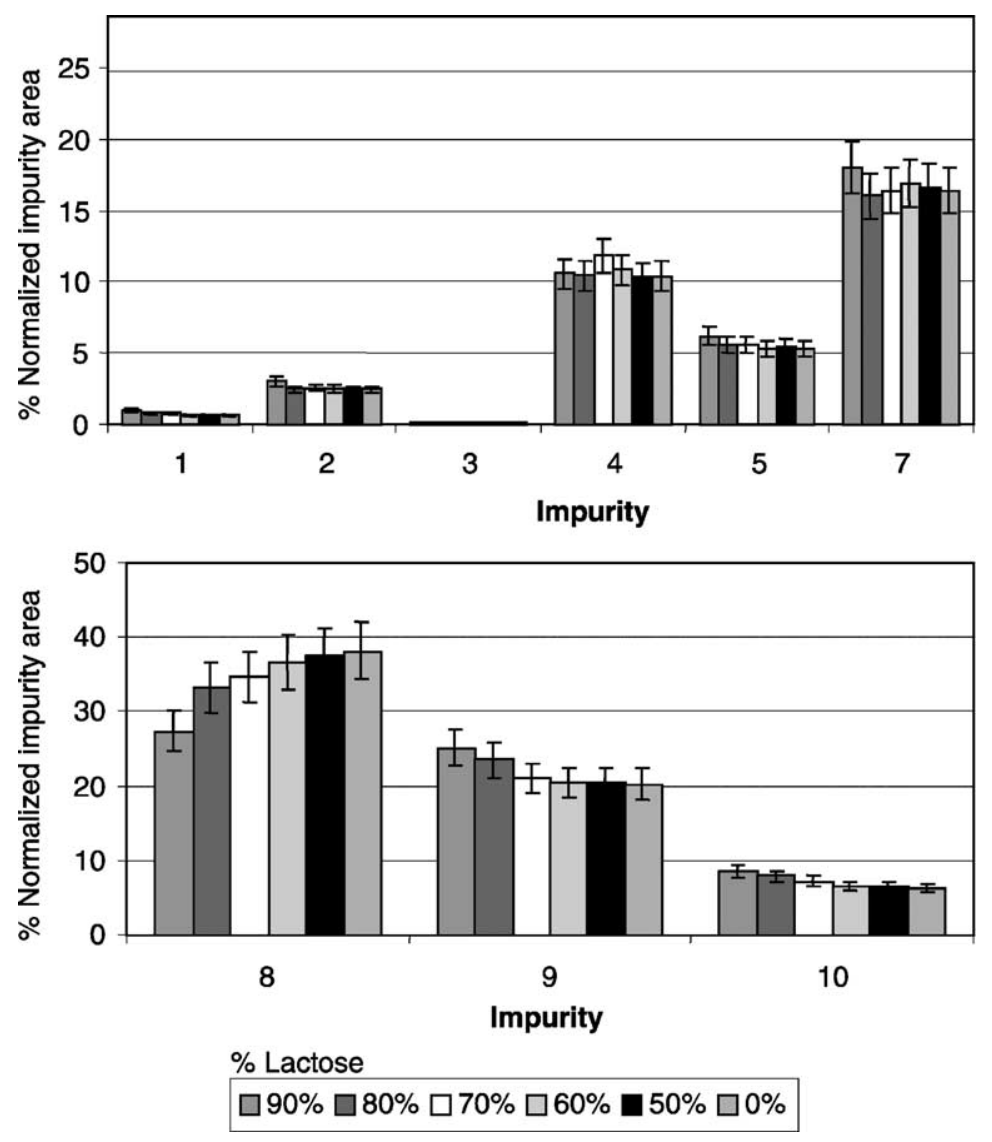

Fig. 11. Influence of the addition of lactose on the impurity extraction (sample RefB).

solvents seem to influence some particular impurities like 2 and 3 for the buffer, 8 and 10 for the extraction solvent. Fortunately, all these variations are unlikely to occur if the solvent dispensers are calibrated and checked on a regular basis.

Table 6

Influence of volume variations (buffer, extraction solvent, recovery solvent)

\begin{tabular}{lllll}
\hline Solvent & $\begin{array}{l}\text { Buffer } \\
\text { volume } \\
(\mathrm{ml})\end{array}$ & $\begin{array}{l}\text { Extraction } \\
\text { volume } \\
(\mathrm{ml})\end{array}$ & $\begin{array}{l}\text { Recovery } \\
\text { volume } \\
(\mu \mathrm{l})\end{array}$ & $\begin{array}{l}\text { Dispenser } \\
\text { accuracy } \\
(\mu \mathrm{l})\end{array}$ \\
\hline First serie buffer & 1.90 & 3.00 & 500 & \pm 20 \\
& $\mathbf{2 . 0 0}$ & & & \\
& 2.10 & & & \pm 15 \\
Second serie & 2.00 & 2.80 & 500 & \\
$\quad$ extraction & & $\mathbf{3 . 0 0}$ & & \pm 3.5 \\
& & 3.20 & & \\
Third serie recovery & 2.00 & 3.00 & 400 & \pm \\
& & & $\mathbf{5 0 0}$ & \\
& & & 600 & \\
\hline
\end{tabular}

Table 7

Influence of volume variations (samples RefA and RefB)

\begin{tabular}{lllll}
\hline Sample & RSD $\%$ & & & \\
\cline { 2 - 5 } & Solvent & Minimum & Maximum & Average \\
\hline RefA & Buffer & 6.1 & 20.8 & 11.5 \\
& Extraction & 3.7 & 18.2 & 10.9 \\
& Recovery & 4.8 & 21.7 & 13.2 \\
RefB & Buffer & 4.9 & 14.5 & 9.2 \\
& Extraction & 4.3 & 13.5 & 7.3 \\
& Recovery & 4.5 & 16.0 & 11.2 \\
\hline
\end{tabular}

\section{Conclusion}

The developed extraction method proved to be repeatable and reproducible. Repeat extractions of the same sample gave an average relative standard deviation of less than $8.5 \%$ within day and less than $10.5 \%$ between days. It was observed that small variations of the MDMA amount (9$11 \mathrm{mg}$ ) gave comparable impurity profiles, and that the most common additive (lactose) did not influence the impurity 
profile if its amount is below 60\%. Most impurities are extracted at $\mathrm{pH} 11.5$ and a higher $\mathrm{pH}$ did not give significant improvements. Diethyl ether seems to better extract low amount impurities $(2,4,5)$ than other solvents like butyl alcohol or toluene, even if butyl alcohol is more interesting for impurity 10 .

A fast nitrogen flow rate means a quick evaporation time but it also leads to a worse precision of the results. Therefore, a slow flow rate has to be preferred.

According to the experiments, large variations of the solvent volumes seem to have a significant influence on the precision of the method. It is then absolutely necessary to use calibrated and reproducible dispensers. Finally, extracts could not be stored more than one day and need to be analyzed the same day they are prepared to avoid impurity degradation.

\section{Acknowledgements}

M. Gimeno thanks Ms Laure Morandat for her technical assistance. The authors are grateful to the financial support from the MILDT (Mission Interministerielle de Lutte contre la Drogue et la Toxicomanie).

\section{References}

[1] Anon., European Union situation report on drug production and drug trafficking 2000-01, Europol, Hague, 2001.

[2] P. Gimeno, F. Besacier, H. Chaudron-Thozet, J. Girard, A. Lamotte, A contribution to the chemical profiling of 3,4methylenedioxymethamphetamine (MDMA) tablets, Forensic Sci. Int. 127 (2002) 1-44.

[3] C. Sten, L. Jonson, N. Artizzu, Factors influencing the extraction of impurities from Leuckart amphetamine, Forensic Sci. Int. 93 (1998) 99-116.

[4] R.J. Renton, J.S. Cowie, M.C.H. Oon, A study of the precursors 3,4-methyenedioxymethylamphetamine and its application to forensic drug analysis, Forensic Sci. Int. 60 (1993) 189-202.

[5] E. Lock, Impurities found in MDMA and MDEA street samples: synthesis, identification and interpretation, in: Proceedings of the ENFSI First European Meeting of
Forensic Science, Lausanne, Switzerland, 17-19 September 1997.

[6] M. Bohn, G. Bohn, G. Blaschke, Synthesis markers in illegally manufactured 3,4-methylenedioxyamphetamine and 3,4-methylenedioxymethamphetamine, Int. J. Legal Med. 106 (1993) 19-23.

[7] A.M. Rashed, R.A. Anderson, L.A. King et al., Solid-phase extraction for profiling of ecstasy tablets, J. Forensic Sci. 45 (2000) 413-417.

[8] D. Stein, The use of heliotrope oil as a precursor source for piperonal, J. Clan. Lab. Invest. Chem. 6 (1996) 17-18.

[9] Drug Enforcement Administration, Uncommon precursor sources used in 3,4-methylenedioxymethamphetamine (MDMA), Microgram 32 (1999) 193-194.

[10] A. Poortman, Unusual manufacturing of MDMA in The Netherlands, J. Clan. Lab. Invest. Chem. 8 (1998) 25-26.

[11] R.R. Laing, B. Dawson, Identification of the major product from the Ritter reaction using safrole, J. Clan. Lab. Invest. Chem. 7 (1997) 22-26.

[12] T.A. Dal Carson, An evaluation of the potential for clandestine manufacture of 3,4-methylenedioxyamphetamine (MDA) analogs and homologs, J. Forensic Sci. 34 (1990) 675-697.

[13] C. Randall Clark, F. Taylor Noggle, J. DeRuiter, GC-MS analysis of products, intermediates and by-products in the synthesis of MDA from isosafrole, Microgram 27 (1994) $188-200$.

[14] T. Lukaszewski, Spectroscopic and chromatographic identification of precursors, intermediates, and impurities of 3,4methylenedioxyamphetamine synthesis, J. Assoc. Off. Anal. Chem. 61 (1978) 951-967.

[15] F.T. Noggle, C. Randall Clarck, J. Deruiter, Gas chromatographic and mass spectrometric analysis of samples from clandestine laboratory involved in the synthesis of ecstasy from sassafras oil, J. Chromatogr. Sci. 29 (1991) 168-173.

[16] A.M.A. Verweij, Clandestine manufacture of 3,4-methylenedioxymethamphetamine (MDMA) by low pressure reductive amination. A mass spectrometric study of some reaction mixtures, Forensic Sci. Int. 45 (1990) 91-96.

[17] A.M.A. Verweij, Impurities in illicit drug preparation (XXIV). Spectroscopic properties of some compounds present in essential oils, used as starting compounds in the synthesis of "designer drugs" of the phenethylamine type, Microgram 28 (1995) 224-228.

[18] A.M.A. Verweij, Impurities in illicit drug preparations: 3,4(methylenedioxy)amphetamine and 3,4-(methylenedioxy)methylamphetamine, Forensic Sci. Rev. 4 (1992) 137-146. 